\title{
LUIS VAISMAN: MEDIO SIGLO DE FORMACIÓN EN HUMANIDADES
}

\author{
Matias Rebolledo Dujisin \\ Universidad de Chile \\ Santiago, Chile \\ majorebo@gmail.com
}

Luis Vaisman (1936-2020) fue un destacado académico, investigador y gestor cultural, de amplia y reconocida trayectoria (casi sesenta años) a nivel nacional. Pero por sobre todas las cosas fue un gran maestro, un formador en el sentido más profundo. Algo de esa trayectoria quisiera esbozar acá. Principalmente, porque esta es la revista de la Facultad donde tantos años ejerció su labor docente y en la que tan activamente participó; pero, también, porque evidentemente la literatura y su enseñanza fue la vocación de su vida. Sin embargo, no es específicamente en el ámbito literario donde me quiero detener, puesto que es, evidentemente, el más reconocido y del que más registros quedan. Para trazar la figura de Vaisman como maestro en Humanidades prefiero enfocarme más en los otros ámbitos donde también dejó su clara huella, mostrando así lo amplio de su formación e influencia: la arquitectura y el teatro. Para esto, me valgo de testimonios de colegas o discípulos en estas disciplinas: Max Aguirre ${ }^{1}$, en arquitectura y Alfredo Castro $^{2}$, colega en

1 Arquitecto por la Universidad de Chile y doctor por la Universidad Politécnica de Madrid, Académico de la Universidad de Chile, y en diversas Universidades: Finis Terrae, Universidad Católica, etc. Los extractos aquí recogidos corresponden al "Prólogo" del libro de Luis Vaisman Hacia una teoría de la arquitectura: antropología arquitectónica (Santiago: Lom, 2015).

2 Me refiero, por supuesto, al director y actor teatral y de cine, fundador del Teatro la Memoria. 
la Escuela Club de Teatro de Fernando González durante poco más de una década. A través de estos testimonios (y anécdotas), pretendo bosquejar el espíritu intelectual, docente y sobre todo de formación de estudiantes, sea del ámbito que fuere, de Luis Vaisman.

Es curioso, pero su llegada a literatura fue relativamente fortuita. Alentado por sus amigos, y con el fin de mejorar su propia escritura, comienza primero a asistir furtivamente a clases (cuenta Max Aguirre que fue cuando realizaba su práctica de arquitectura, supervisando obras que realizaban en nuestra Facultad), para luego entrar por admisión especial, con su licenciatura de arquitecto en la Universidad de Chile ${ }^{3}$. Pronto abandona la escritura por la que, sin duda, fue su verdadera vocación: la enseñanza. Son años donde recibiría la importante influencia de sus dos grandes maestros, Félix Martínez Bonatti y Jorge Guzmán, además de compartir su formación con dos grandes amigos (y sendos maestros en la Universidad de Chile), Lucía Invernizzi y Federico Schopf ${ }^{4}$. En 1962 o 63 ya era ayudante de Félix Martínez, quien le daría la única instrucción a principios de año, en el curso de Estilística, antecedente de la teoría literaria: "Usted Luis, va a leer el Ulises con los alumnos", tarea que le tomó todo el año (los cursos eran anuales). Eran los años dorados del (antiguo) Pedagógico: Goic, Rabanales, los dos profesores antes mencionados, etc.

Hay que señalar, también, que la formación en esos años era bastante más intensa que hoy en día: la carrera duraba entre cinco y seis años, la formación incluía una buena base en filosofía, historia, o historia del arte, las tesis eran verdaderas tesis de magíster. De esa "camada" saldrían, como señalé en nota, dos prestigiosos artistas y académicos, mucho más reconocidos internacionalmente: Dorfman y Kay. Pero Vaisman se quedó en la Chile, aun cuando trabajó en otras universidades, en Valparaíso y en Valdivia. Pero, mientras estaba estudiando literatura, paralelamente se convirtió en profesor de la FAU, estableciendo este vínculo ya por siempre.

\footnotetext{
Como me contara alguna vez, renunció a la empresa grande de arquitectura donde ya trabajaba, cambió su "auto largo" por una motoneta y se dedicó a hacer clases particulares de lenguaje para dedicarse completamente a la carrera.

Que, en realidad, habían entrado un año antes; de su propia generación los únicos que optaron por la licenciatura -la mayoría entraba por pedagogía-, y por ende sus compañeros directos, fueron Ronald Kay y Ariel Dorfman.
} 
De su trayectoria académica no se puede destacar sus investigaciones o publicaciones por su número, pero sí por su relevancia ${ }^{5}$. Amado u odiado, lo cierto es que todos los que alguna vez tuvimos clases con él las recordamos, aunque más no sea por su performance. O la risa "fuera de lugar", o el nivel de erudición. Pero, por sobre todo -destaco esto a título personal, tanto como alumno cuanto como ayudante-, porque siempre iba más allá -del texto, de la teoría, de la materia-, donde algo pasaba, algo hacía un clic, que nos cambiaba la forma de ver o entender el texto, o la literatura, o incluso la vida ${ }^{6}$. Y siempre estaba un poco más acá o más allá: cuando ya todos se habían convertido al posestructuralismo o eran arrastrados por la postmodernidad, con mayor ahínco se dedicaba a enseñar la Poética de Aristóteles ${ }^{7}$. Cuando nadie "respetable" hablaba seria y académicamente de la Ciencia Ficción (hace cuarenta años), publicaba complejos artículos sobre el género y hacía cursos donde traía -literalmente- una caja con los libros que los alumnos tenían que leer. Dictó, también, el primer curso específico de literatura homoerótica en el Departamento. Etcétera.

No fuimos muchos los discípulos de Vaisman, pero -y esto es lo que quiero destacar-, quienes lo fuimos, lo fuimos de manera integral, bajo un modelo de formación y docencia que hoy ya prácticamente ha desaparecido. Ser su ayudante no significaba hacerle sus clases y corregirle pruebas; implicaba aprender de literatura, de cultura, de la docencia (en su sentido práctico pero sobre todo ético), de valores, de la vida. Significaban conversaciones eternas e inagotables, en la Universidad, en el café, en su casa, en largos paseos. Era una formación humana e integral que es casi imposible de replicar ahora. Los testimonios a continuación, en estas otras disciplinas, buscan precisamente

5 La editorial LOM publicará este 2021 una selección de sus producciones, bastante dispersas. Algunas de estos artículos pertenecen a esta Revista: "La obra dramática: un concepto operacional para su análisis e interpretación en el texto" ( $\left.\mathrm{N}^{\circ} 14\right)$; "La estructura del cuento en Kafka" ( $\left.\mathrm{N}^{\circ} 22\right)$; "En torno a la ciencia-ficción: propuesta para la descripción de un género histórico" ( $\left.\mathrm{N}^{\circ} 25\right)$; "El concepto de 'espectáculo' en el arte poética de Aristóteles" ( $\left.\mathrm{N}^{\circ} 72\right)$.

${ }^{6} \quad$ El poeta y editor Juan Manuel Silva (fundador de Montacerdos) recordó a propósito de su muerte, algunas de las frases que lo habían marcado, para siempre, de sus clases. También aseguró que nunca se hubiera convertido en editor si no fuera por las correcciones de Vaisman a sus trabajos. Recuerdos y, sobre todo, de anécdotas como esa están plagadas las redes, sobre todo a partir de su reciente muerte.

Exasperantes cursos de Estética, que consistían exclusivamente en la lectura atenta de la Poética, y que no lograban pasar de la mitad del texto en el semestre completo. 
eso: ampliar la mirada sobre su figura académica, hoy tan centrada en el número de publicaciones y proyectos adjudicados.

Como señalaba anteriormente, durante el tiempo en que estudiaba y luego era ayudante de literatura, fue paralelamente profesor de la FAU, en nuestra Universidad. De esos años nos cuenta Max Aguirre en el "Prólogo" de Hacia una teoría de la arquitectura: "Desde el primer día [como estudiante] me impresionó y aprecié la calidad de sus clases [...]. Sus cursos estaban habitualmente al tope [...]. Una cualidad notable, especialmente en el contexto de las tecnologías para hacer clases aplicadas en la actualidad, era su explicación ininterrumpida, coherente y clara que envolvía y capturaba nuestra atención sin soltarla hasta el término de la sesión" (9). Es cierto que con el tiempo fue perdiendo esa habilidad para ir del principio al fin, incluyendo numerosas digresiones, de manera cerrada y perfecta, pero esa también fue una decisión pedagógica: alguna vez, hablando con algún alumno de confianza, le preguntó por qué no le hacían preguntas en clases, a lo que este respondió que "sus clases están demasiado bien armadas, no queda espacio por donde entrar"; desde allí en adelante el dejar cabos sueltos, hacer digresiones que no necesariamente se cerraban fue, por así decirlo, también una estrategia.

Lo importante de su papel en la formación de arquitectos de la Universidad de Chile no se agota en la calidad de sus clases, ni mucho menos. Se trata de un profesor de teoría arquitectónica (rápidamente se dio cuenta de que lo suyo no estaba en la maqueta y el cálculo estructural) que instaló una nueva forma de comprender la arquitectura, que irían a seguir sus discípulos, como Boris Benado, e indirectamente Max Aguirre: "lo que con el tiempo más he valorado [...] es la marca indeleble que me dejó su formación humanista y el rol humanizante que le cabe al arquitecto y a la arquitectura en la sociedad. Cualquier contenido expuesto por él trascendía la mera fruición del erudito para llevar a quien lo escuchara ante la pregunta clave en la que se encontraba el nudo de la cuestión [...]. Adquiría pleno sentido la necesidad de la historia y la teoría para la formación del arquitecto, aunque era evidente que este conocimiento no era del tipo productivo que hace del saber una herramienta que encuentra su directa aplicación en las tareas que generan, por ejemplo, el proyecto. En cambio, iba construyendo imperceptiblemente en el estudiante un acervo con el cual discernir la realidad cualquiera fuera la circunstancia en que se desenvolvieran los avatares de la profesión" (9).

La verdad es que su aporte en el campo de la arquitectura es significativo, lo que se evidencia en las dos publicaciones que hiciera la editorial LOM 
en 2015 y $2018^{8}$, y el impacto que estas tuvieron. Luego de su seminario de investigación, sobre el gótico y bajo la dirección de José Ricardo Morales, comienza a hacer clases de Historia de la cultura, en Arquitectura, en 1963, y en 1964 de Teoría de la arquitectura. Hasta que se trasladara definitivamente a Filosofía y Humanidades durante la dictadura, continuó haciendo clases en estos cursos e investigando en estos ámbitos. Alcanzaría a publicar en 1972 Semiología arquitectónica, un texto pionero en la materia tanto en Chile como en Latinoamérica9. Su tesis, terminada en ese mismo 1972, fue Hacia una teoría de la arquitectura. El devenir de esa tesis tiene una historia "policial". En septiembre del 73 un funcionario de la FAU le avisó que estaban quemando los libros y le ofreció robarse las tres copias empastadas de dicha tesis. Una de ellas se perdió, otra se la quedó Vaisman, y la tercera quedó con Boris Benado, su entonces ayudante. Resultó que ese texto, que Vaisman daba por perdido, fue ampliamente difundido en fotocopias por Benado en la Escuela Nacional Superior de Arquitectura y Paisaje de Bordeaux por más de veinte años, y luego, desde el 2000 en la Universidad de Chile. De este modo, ese libro rescatado y pirateado fue la base de la formación en teoría arquitectónica tanto en Francia como en la Universidad de Chile, durante décadas ${ }^{10}$. Casi cincuenta años después de su publicación, sus aportes a la teoría arquitectónica (a la que dedicó apenas sus diez primeros años de su vida académica) siguen siendo vigentes y fundamentales.

Al mundo del teatro entró también relativamente por azar. O bien, como una decisión consciente y estratégica: una forma de encontrar un "nicho ecológico", donde aún no se había desarrollado plenamente la teoría, al menos en Chile. De este modo, junto a Nelly Donoso y Eduardo Thomas fueron los especialistas en teatro de la Facultad por varias décadas. Tal vez su artículo más relevante y más veces citado sea "La obra dramática: un concepto operacional para su análisis e interpretación en el texto", publicado en 1979, en el número 14 de esta Revista. Pero más allá de sus aportes a la Academia, me interesa relevar su papel en el teatro no solo por sus estudios

\footnotetext{
El otro texto fue Semiología Arquitectónica. Una presentación. Santiago: LOM Ediciones, 2018.

$9 \quad$ Es el texto que LOM reeditó, y que hasta hoy, casi cincuenta años después, sigue siendo uno de los poquísimos trabajos en la materia y un referente a nivel nacional.

10 Descargando algunos de los programas disponibles en internet, pude comprobar que incluso en los últimos años seguía siendo la bibliografía única o fundamental de los cursos de teoría en la FAU, tanto en su versión fotocopiada como, a partir de 2016, en el libro de LOM.
} 
en nuestro ámbito, sino en la formación de actores. En la década de los 90 y en la primera de los 2000 fue profesor y jefe docente de la Escuela Club de Teatro, de Fernando González, que tiene en su historial la formación de un impresionante listado de actores y actrices a nivel nacional.

Al menos por una década fueron compañeros en la docencia de dicha Escuela con Alfredo Castro, a quien pedí que me relatara algunas impresiones de su rol en la formación de actores ${ }^{11}$. Más allá del evidente cariño y respeto que manifiesta, y de señalar que, como es lugar común, "los alumnos lo adoraban", lo recuerda siempre como una persona de un saber inmenso, pero que sobre todo era muy generoso con dicho saber: "en los exámenes, escuchar los comentarios y críticas a los estudiantes era siempre una lección". Cabe decir que en el Club de Teatro se han formado no solo importantes actores, sino que también destacados directores teatrales contemporáneos, o profesores de teatro: Rodrigo Pérez, Cristián Plana, Carlos Bórquez, etc. De ahí que las clases de historia del teatro o de semiótica hayan sido también muy relevantes en dicha institución ${ }^{12}$.

Señala Alfredo Castro, por ejemplo, que "con los ejercicios que hacíamos con los estudiantes, que eran muy radicales, muchas veces, él tenía una apertura y una proximidad a lo supuestamente 'nuevo' o 'distinto', que era muy fascinante, porque inmediatamente nos llamaba un poco a terreno, diciendo 'miren, esto ya se hizo el año tanto, en Alemania, en Francia, en Bélgica, en donde fuera, y lo hizo tal persona, y terminó en tal cosa'. Por eso era siempre un aporte. Un aporte a no pensar nunca que uno estaba inventando algo rigurosamente 'nuevo', 'trasgresor', que todo ya se había hecho". Aclarando que no es ni broma ni exageración, no duda en afirmar que "termina una parte de la historia del teatro de Chile", ya que "él fue alguien muy, muy importante para varias generaciones, de gente notable que siguió este camino".

11 Los textos aquí citados son transcripciones de conversaciones privadas, por lo que no tienen referencia.

12 Como señala Alfredo Castro, fue Vaisman el que trajo la semiótica a la Escuela. Castro era un actor, director e investigador ya absolutamente consagrado, pero todo este 'saber' sobre teatro era algo nuevo, y que no conocía. Por ende le pidió asistir a sus clases, a lo que Vaisman contestó que "por ningún motivo, porque esto no te va a servir para nada; va a pasar el tiempo y va a ser algo muy muy específico para algunas personas, pero para los creadores como tú esto no te va a servir, y puede que incluso sea un crimen contra la creación". 
Cabe señalar, por último, que quien fuera su ayudante en esos años en la Escuela, César Farah, es hoy en día académico de la Escuela de Teatro de la Universidad de Chile. De este modo, y sin mencionar el número infinito de alumnos y alumnas, tesistas, o ayudantes, para los que fue parte importante de su formación, somos muchos los que nos podemos considerar discípulos directos de este Maestro, maestro en el mundo de la arquitectura, del teatro, y por supuesto de la literatura. Dejo fuera, a su vez, otros aspectos igualmente relevantes de su trayectoria, como los años en la jibarizada Facultad en La Reina; su rol como Director Académico en el decanato de Lucía Invernizzi, donde, entre otras cosas, les tocó repatriar a los profesores exiliados (Leonidas Morales, Kemy Oyarzún, Federico Schopf, etc.) o su participación en la reforma educacional o la fundación de la SOCHEL. 